\title{
Model Independent Naturalness Bounds on Magnetic Moments of Majorana Neutrinos
}

\author{
Mikhail Gorchtein, Nicole F. Bell, Michael J. Ramsey-Musolf, Petr Vogel \\ and Peng Wang
}

California Institute of Technology, Pasadena, CA 91125, USA

\begin{abstract}
We analyze the implications of neutrino masses for the magnitude of neutrino magnetic moments. By considering electroweak radiative corrections to the neutrino mass, we derive modelindependent naturalness upper bounds on neutrino magnetic moments, generated by physics above the electroweak scale. For Majorana neutrinos, these bounds are weaker than present experimental limits if $\mu_{v}$ if generated by new physics at $\sim 1 \mathrm{TeV}$, and surpass current experimental sensitivity only for new physics scales $>10-100 \mathrm{TeV}$. The discovery of a neutrino magnetic moment near present limits would thus signify that neutrinos are Majorana particles.
\end{abstract}

Keywords: neutrino magnetic moment, majorana

PACS: 12.15.-y 12.15.Lk 12.60.-i 13.15.+g 14.60.Pq

In the Standard Model (minimally extended to include non-zero neutrino mass) the neutrino magnetic moment is given by $\mu_{v} \approx 3 \times 10^{-19}\left(\frac{m_{v}}{1 \mathrm{eV}}\right) \mu_{B}$ [1]. An experimental observation of a larger magnetic moment would be an unequivocal indication of physics beyond the minimally extended Standard Model. Current laboratory limits, $\mu_{v}<1.5 \times 10^{-10} \mu_{B}[2]$ and $\mu_{v}<0.7 \times 10^{-10} \mu_{B}$ [3], are obtained from solar and reactor experiments, respectively. Slightly stronger bounds are obtained from astrophysics, $\mu_{v}<3 \times 10^{-12}$ [4]. In the case of Dirac neutrinos, the neutrino mass constrains the magnetic moments to be [5] $\mu_{v} \leq 10^{-15} \mu_{B}$, which is several orders of magnitude more stringent than current experimental constraints.

Following Refs. [5, 6] we assume that the magnetic moment is generated by unspecified physics beyond the SM at an energy scale $\Lambda$ above the electroweak scale. We shall work exclusively with dimension $D>4$ operators that involve only SM fields, obtained by integrating out the physics above the scale $\Lambda$, and respect the $S U(2)_{L} \times U(1)_{Y}$ symmetry of the SM, and contains only SM fields charged under these gauge groups. The lowest order contributions to the neutrino (Majorana) mass arise from the usual five and seven dimensional operators containing Higgs and lefthanded lepton doublet fields, $L$ and $H$, respectively, $\left[\mathbf{O}_{\mathbf{M}}^{\mathbf{5 D}}\right]_{\alpha \beta}=\left(\overline{\mathbf{L}_{\alpha}^{\mathbf{c}}} \varepsilon \mathbf{H}\right)\left(\mathbf{H}^{\mathbf{T}} \varepsilon \mathbf{L}_{\beta}\right)$ the five dimensional, and $\left[\mathbf{O}_{\mathbf{M}}^{7 \mathrm{D}}\right]_{\alpha \beta}=\left(\overline{\mathbf{L}_{\alpha}^{\mathbf{c}}} \varepsilon \mathbf{H}\right)\left(\mathbf{H}_{\varepsilon}^{\mathbf{T}} \mathbf{L}_{\beta}\right)\left(\mathbf{H}^{\dagger} \mathbf{H}\right)$ the seven dimensional one. We use the notation $\varepsilon=-i \tau_{2}, \overline{L^{c}}=L^{T} C, C$ denotes charge conjugation, and $\alpha, \beta$ are flavor indices. The neutrino magnetic moment operator is generated by dimension 7 operators involving the $S U(2)_{L}$ and $U(1)_{Y}$ gauge fields, $W_{\mu}^{a}$ and $B_{\mu}$, respectively, $\left[\mathbf{O}_{\mathbf{B}}\right]_{\alpha \beta}=\mathbf{g}^{\prime}\left(\overline{\mathbf{L}}_{\alpha} \boldsymbol{\varepsilon} \mathbf{H}\right) \sigma^{\mu v}\left(\mathbf{H}^{\mathbf{T}} \boldsymbol{\varepsilon} \mathbf{L}_{\beta}\right) \mathbf{B}_{\mu v}$, and $\left[\mathbf{O}_{\mathbf{W}}\right]_{\alpha \beta}=\mathbf{g}\left(\overline{\mathbf{L}}_{\alpha}^{\mathbf{c}} \varepsilon \mathbf{H}\right) \sigma^{\mu v}\left(\mathbf{H}^{\mathbf{T}} \boldsymbol{\varepsilon} \boldsymbol{\tau}^{\mathbf{a}} \mathbf{L}_{\beta}\right) \mathbf{W}_{\mu v}^{\mathbf{a}}$. In the above definitions, $B_{\mu v}=$ $\partial_{\mu} B_{v}-\partial_{v} B_{\mu}$ and $W_{\mu \nu}^{a}=\partial_{\mu} W_{v}^{a}-\partial_{v} W_{\mu}^{a}-g \varepsilon_{a b c} W_{\mu}^{b} W_{v}^{c}$ are the $\mathrm{U}(1)_{Y}$ and $\mathrm{SU}(2)_{L}$ are the 
field strength tensors, respectively, and $g^{\prime}$ and $g$ are the corresponding couplings. The three 7D operators defined above do not form a basis under renormalization. The full basis of 7D operators may be found in [7].

The operator $O_{W}$ is asymmetric in flavor, and it is useful to express it in terms of operators with explicit flavor symmetry, $\left[O_{W}^{ \pm}\right]_{\alpha \beta}=\frac{1}{2}\left(\left[O_{W}\right]_{\alpha \beta} \pm\left[O_{W}\right]_{\beta \alpha}\right)$. Operators $O_{M}^{5 D}, O_{M}^{7 D}$ and $O_{W}^{+}$are flavor symmetric, while $O_{B}$ and $O_{W}^{-}$are antisymmetric. Our effective Lagrangian is therefore

$$
\mathscr{L}=\frac{C_{M}^{5 D}}{\Lambda} O_{M}^{5 D}+\frac{C_{M}^{7 D}}{\Lambda^{3}} O_{M}^{7 D}+\frac{C_{B}}{\Lambda^{3}} O_{B}+\frac{C_{W}^{+}}{\Lambda^{3}} O_{W}^{+}+\frac{C_{W}^{-}}{\Lambda^{3}} O_{W}^{-}+\ldots,
$$

where the "+ $+\cdots "$ denote other terms that are not relevant to the present analysis. After spontaneous symmetry breaking, the above operators reduce to the flavor symmetric Majorana mass term $\frac{1}{2}\left[\mathbf{m}_{v}\right]_{\alpha \beta} \overline{v^{\mathbf{c}}}{ }_{\alpha} v_{\beta}$, and flavor antisymmetric Majorana magnetic moment interaction $\frac{1}{2}\left[\mu_{v}\right]_{\alpha \beta}{\overline{v^{\mathbf{c}}}}_{\alpha} \sigma^{\mu v} v_{\beta} \mathbf{F}_{\mu v}$, where $F_{\mu v}$ is the electromagnetic field strength tensor. At the EW scale, we have

$$
\begin{aligned}
\frac{\left[\mu_{v}\right]_{\alpha \beta}}{\mu_{B}} & =\frac{2 m_{e} v^{2}}{\Lambda^{3}}\left(\left[C_{B}\left(M_{W}\right)\right]_{\alpha \beta}+\left[C_{W}^{-}\left(M_{W}\right)\right]_{\alpha \beta}\right), \\
\frac{1}{2}\left[m_{v}\right]_{\alpha \beta} & =\frac{v^{2}}{2 \Lambda}\left[C_{M}^{5 D}\left(M_{W}\right)\right]+\frac{v^{4}}{4 \Lambda^{3}}\left[C_{M}^{7 D}\left(M_{W}\right)\right],
\end{aligned}
$$

where the Higgs VEV is $\left\langle H^{T}\right\rangle=(0, v / \sqrt{2})$.

Below, we calculate radiative corrections to the neutrino mass operators $\left(O_{M}^{5 D}\right.$ and $O_{M}^{7 D}$ ) generated by the magnetic moment operators $O_{W}^{-}$and $O_{B}$ and put constraints on the size of the magnetic moment in terms of the neutrino mass, using Eqs.(2) and (3).

\section{- 5D mass term - 1 loop}

One-loop radiative corrections only yield a contribution to $O_{M}^{5 D}$ associated with the flavor symmetric operator $O_{W}^{+}$. No model independent bound can be obtained from the oneloop radiative corrections from magnetic momentto the 5D mass operator.

\section{- 7D mass term - one loop}

As the operator $O_{W}^{-}$is flavor antisymmetric, it must be multiplied by another flavor antisymmetric contribution in order to produce a flavor symmetric mass term. This can be accomplished through insertion of Yukawa couplings in the diagram shown in Fig. 1 [6]. This diagram provides a logarithmically divergent contribution to the 7D

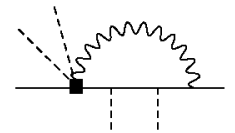

FIGURE 1. Contribution of $O_{W}^{-}$to the 7D neutrino mass operator.

mass term, given by

$$
\left[C_{M}^{7 D}\left(M_{W}\right)\right]_{\alpha \beta} \simeq \frac{3 g^{2}}{16 \pi^{2}} \frac{m_{\alpha}^{2}-m_{\beta}^{2}}{v^{2}} \ln \frac{\Lambda^{2}}{M_{W}^{2}}\left[C_{W}^{-}(\Lambda)\right]_{\alpha \beta}
$$


where $m_{\alpha}$ are the charged lepton masses, and the exact coefficient has been computed using dimensional regularization and renormalized with modified minimal subtraction. Using this result, as well as Eqs. (2) and (3), leads to bound (i) in Table 1.

If we insert $O_{B}$ in the diagram in Fig. 1, the contribution vanishes. A non-zero contribution to $O_{M}^{7 D}$ from $O_{B}$ can arise at two-loop level, for instance, from a virtual $W$ boson loop as in Fig. 2 [6]. This mechanism gives the leading contribution of the operator

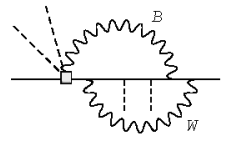

FIGURE 2. Representative contribution of $O_{B}$ to the 7D neutrino mass operator at two loop order.

$O_{B}$ to the 7D mass term. The $O_{B}$ and $O_{W}$ contributions to the 7D mass term are thus related by $\frac{\left(\delta m_{v}\right)^{B}}{\left(\delta m_{v}\right)^{W}} \approx \frac{\alpha}{4 \pi} \frac{1}{\cos ^{2} \theta_{W}}$, where $\theta_{W}$ is the weak mixing angle. The corresponding limit is shown as bound (iii) in Table 1 .

\section{- 5D mass term - two loop}

The neutrino magnetic moment operator $O_{W}^{-}$will also contribute to the $5 \mathrm{D}$ mass operator via two-loop matching of the effective theory onto the full theory at at the scale $\Lambda$, as shown in Fig. 3. This diagram contributes to the 5D mass operator, and we provide a

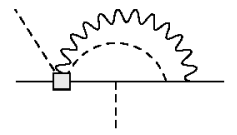

FIGURE 3. Representative contribution of $O_{W}^{-}$and $O_{B}$ to the 5D neutrino mass operator. naïve dimensional analysis [8] (NDA) estimate for the $O_{B}$ and $O_{W}$ insertions:

$$
\left[C_{M}^{5 D}(\Lambda)\right]_{\alpha \beta} \simeq \frac{g^{2}}{\left(16 \pi^{2}\right)^{2}} \frac{m_{\alpha}^{2}-m_{\beta}^{2}}{v^{2}}\left[C_{W}^{-}(\Lambda)\right]_{\alpha \beta} .
$$

Using Eqs.(2) and (3), we obtain the bound (ii) in Table 1. The $O_{B}$ contribution to the $5 \mathrm{D}$ mass term is the same as that for $O_{W}$, except for a factor of $\left(g^{\prime} / g\right)^{2}=\tan ^{2} \theta_{W}$, and it corresponds to the bound (iv) in Table 1. In doing so, we have neglected the running of the operator coefficients from the scale $\Lambda$ to $M_{W}$ since the effects are higher order in the gauge couplings and have a negligible numerical impact on our analysis. Our conclusions can be summarized according to the scale of the new physics, $\Lambda$ :

I) $\Lambda \leq 10 \mathrm{TeV}$

- No conflict with experimental limits.

- Both $O_{W}$ and $O_{B}$ contributions to $\mu_{v}$ are possible, though $O_{W}$ contributions are more tightly constrained.

II) $\Lambda \geq 10 \mathrm{TeV}$

- $\mu_{\tau \mu}, \mu_{\tau e}$ bounds stronger than experimental limits

- $\mu_{\mu e}$ bound weaker than experimental limits 
TABLE 1. Summary of constraints on the magnitude of the magnetic moment of Majorana neutrinos. The upper two lines correspond to a magnetic moment generated by the $O_{W}$ operator, while the lower two lines correspond to the $O_{B}^{-}$operator. $R_{\alpha \beta}=$ $\frac{m_{\tau}^{2}}{\left|m_{\alpha}^{2}-m_{\beta}^{2}\right|}$, with $m_{\alpha}$ being the masses of charged lepton masses. Numerically, $R_{\tau e} \simeq R_{\tau \mu} \simeq 1$ and $R_{\mu e} \simeq 283$.

\begin{tabular}{l|l|l}
\hline i) 1-loop, 7D & $\mu_{\alpha \beta}^{W}$ & $\leq 1 \times 10^{-10} \mu_{B}\left(\frac{\left[m_{v}\right]_{\alpha \beta}}{1 \mathrm{eV}}\right) \ln ^{-1} \frac{\Lambda^{2}}{M_{W}^{2}} R_{\alpha \beta}$ \\
ii) 2-loop, 5D & $\mu_{\alpha \beta}^{W}$ & $\leq 1 \times 10^{-9} \mu_{B}\left(\frac{\left[m_{v}\right]_{\alpha \beta}}{1 \mathrm{eV}}\right)\left(\frac{1 \mathrm{TeV}}{\Lambda}\right)^{2} R_{\alpha \beta}$ \\
\hline iii) 2-loop, 7D & $\mu_{\alpha \beta}^{B}$ & $\leq 1 \times 10^{-7} \mu_{B}\left(\frac{\left[m_{v}\right]_{\alpha \beta}}{1 \mathrm{eV}}\right) \ln ^{-1} \frac{\Lambda^{2}}{M_{W}^{2}} R_{\alpha \beta}$ \\
iv) 2-loop, 5D & $\mu_{\alpha \beta}^{B}$ & $\leq 4 \times 10^{-9} \mu_{B}\left(\frac{\left[m_{v}\right]_{\alpha \beta}}{1 \mathrm{eV}}\right)\left(\frac{1 \mathrm{TeV}}{\Lambda}\right)^{2} R_{\alpha \beta}$ \\
\hline
\end{tabular}

- Same limit irrespective of whether $\mu_{v}$ generated by $O_{W}$ and $O_{B}$ III) $\Lambda \geq 100 \mathrm{TeV}$

- The $\mu_{\alpha \beta}$ bound becomes stronger than current experimental constraints, for all $\alpha, \beta$.

We emphasize that the bound (iv) in Table 1 is the most general bound on the magnetic moment.

We thank Vincenzo Cirigliano, Concha Gonzalez-Garcia, and Mark Wise for illuminating conversations. This work was supported in part under U.S. DOE contracts DEFG02-05ER41361 and DE-FG03-92ER40701.

\section{REFERENCES}

1. W. J. Marciano and A. I. Sanda, Phys. Lett. B 67, 303 (1977); B. W. Lee and R. E. Shrock, Phys. Rev. D 16, 1444 (1977); K. Fujikawa and R. Shrock, Phys. Rev. Lett. 45, 963 (1980).

2. J. F. Beacom and P. Vogel, Phys. Rev. Lett. 83, 5222 (1999); D. W. Liu et al., Phys. Rev. Lett. 93, 021802 (2004).

3. H. T. Wong [TEXONO Collaboration], hep-ex/0605006. B. Xin et al. [TEXONO Collaboration], Phys. Rev. D 72, 012006 (2005); Z. Daraktchieva et al. [MUNU Collaboration], Phys. Lett. B 615, 153 (2005).

4. G.G. Raffelt, Phys. Rep. 320, 319 (1999).

5. N. F. Bell, V. Cirigliano, M. J. Ramsey-Musolf, P. Vogel and M. B. Wise, Phys. Rev. Lett. 95, 151802 (2005).

6. S. Davidson, M. Gorbahn and A. Santamaria, Phys. Lett. B 626, 151 (2005).

7. N.F. Bell, M. Gorchtein, M.J. Ramsey-Musolf, P. Vogel, P. Wang, Phys. Lett. B it in press, arXiv: [hep$\mathrm{ph} / 0606248]$

8. C. P. Burgess and D. London, Phys. Rev. D 48, 4337 (1993).

9. D. N. Spergel et al., astro-ph/0603449. 